\title{
Multiple agents in biological control: improving the odds?
}

\author{
Madlen Denoth, ${ }^{*}$ Leonardo Frid, and Judith H. Myers \\ Department of Zoology, University of British Columbia, 6270 University Boulevard, Vancouver, BC, Canada V6T 1Z4
}

Received 29 January 2001; accepted 4 December 2001

\begin{abstract}
The current interest in risks associated with classical biological control led us to review the literature to determine whether the introduction of multiple biological control agents has been more effective than the introduction of a single control agent. We analyzed 59 projects against weeds and 108 projects against insect pests. Establishment of control agents was significantly higher in single-agent projects than in multiple-agent projects against insect pests, but not in projects against weeds. The success of biological control against weeds increased with the number of agents released. However, no relationship was found between the number of agents released and biological control success for insect pests. These results suggest that negative interactions may play a significant role amongst biological control agents of insects. Thus, multiple releases may be effective against weeds only. In over $50 \%$ of the successful multiple-agent projects against weeds and insect pests, a single agent was shown to be responsible for success. This result indicates that, in a majority of biological control projects, multiple agents are not released for a cumulative control effect but to increase the likelihood that the right control species is released (lottery model). Considering the possibility of negative interactions among biological control agents and taking into account the risks associated with natural enemy introductions, we recommend restraint in the introduction of multiple agents for biological control. @ 2002 Elsevier Science (USA). All rights reserved.
\end{abstract}

Keywords: Biological control; Competitive exclusion; Establishment rates; Success rates; Multiple agents

\section{Introduction}

In recent years, classical biological control has come under increasing scrutiny for its nontarget effects (Cory and Myers, 2000; Hawkins and Marino, 1997; Howarth, 1991). However, there are many examples of successful biological control (Bellows, 2001; Caltagirone, 1981; McFadyen, 1998), and the need for biological control is increasing (Cory and Myers, 2000; Hawkins, 1993; McFadyen, 1998). Taking into account both the risks associated with biological control and the necessity for its practice, it is suggested that rather than eliminate biological control altogether, we should minimize the number of natural enemy introductions.

When tackling a particular pest problem with biological control, one must determine how many agents may be necessary for successful control. This question

\footnotetext{
${ }^{*}$ Corresponding author. Fax: +604-822-2416.

E-mail addresses: denoth@zoology.ubc.ca (M. Denoth), frid@ zoology.ubc.ca (L. Frid), myers@zoology.ubc.ca (J.H. Myers).
}

has been asked in the past (Myers, 1985; Myers et al., 1989). Both of these studies showed that in examples of successful biological control involving introductions of multiple agents, most often only a single agent was considered responsible for the success. Rather than imposing cumulative stress on the pest by using multiple agents, these projects have treated multiple agents as "lottery tickets" to success (Myers, 1985). The higher the number of agents introduced, the more likely it is that the right one will be amongst them. In some cases, however, using multiple agents may actually reduce the likelihood of success through the process of competitive exclusion (Ehler and Hall, 1982).

Given the recent publication of extensive databases documenting biological control projects for both weeds (Julien and Griffiths, 1998) and arthropod pests (Greathead and Greathead, 1992), and the acknowledgment of the risks associated with introducing unnecessary agents, we revisit the question: "How many insects are necessary for the biological control of weed and insect pests?" Specifically, we ask the following 
questions: (1) How does the number of agents introduced influence establishment rates? (2) Does using multiple vs. single agents increase the rates of success of biological control? (3) In projects involving multiple agents, how many agents are actually responsible for success?

\section{Methods}

\subsection{Weeds}

The data for our analysis of weed biological control were taken from List A in Julien and Griffiths (1998), a compilation of information on biological control projects against weeds throughout the world in which exotic insects and fungi were used as biological control agents. For the purpose of this study, we define a project as the release of one or more biological control agents against one weed species in a specific geographical area, usually a country. Due to inconsistent definitions of success by different researchers and due to differences in the methods used to determine the success of the projects, there may be disagreement concerning the level of success of certain programs. To minimize variability in the data set, we chose conservatively, selecting only biological control projects which were either unsuccessful or clearly successful, or for which a decline in the density of the weed was reported. Releases of control agents since 1990 for which success was not documented were not included because it may be too early for evaluation. In some cases, there was insufficient information available to determine the success due to a control agent, or disturbances to the release sites made an evaluation of the performance of a control agent impossible. These projects were not considered for analysis. If there were several projects in which one or several of the same control agents were involved in success, we chose the project that was started first to reduce sampling bias because it is possible that knowledge from an earlier project led to success in later ones.

If several projects against different weeds involving the same control agents started in the same year, we chose the most comprehensive program (e.g., project against Opuntia stricta (Haworth) Haworth var. stricta (Haworth) Haworth in Australia chosen out of several control projects against different Opuntia species). Control agents that were originally considered to be successful, but failed later, were not recorded as being involved in success (e.g., Chelidinea tabulata (Burmeister) released against Opuntia stricta var. stricta in Australia). When counting the control agents introduced for a project, we did not include agents that slightly attacked a weed, but were not originally released against the weed.
Out of the 59 selected biological control projects against weeds, 19 involved the release of one agent only, 16 projects used two, 8 projects used three, 6 projects used four, and 10 projects used five or more agents. Thirty-three projects were successful and 28 of these projects involved the use of multiple agents; 26 were unsuccessful (Table 1).

\subsection{Insect pests}

Data on biological control of insects were taken from the BIOCAT database of biological control projects (Greathead and Greathead, 1992). The data were collected as follows. First, an arbitrary number of successful and unsuccessful projects were chosen at random from the database. As with the weeds, we then reduced bias in the data by selecting the earliest successful projects against the chosen pests. For unsuccessful projects, the randomly selected projects were used. Out of the 64 successful and 44 unsuccessful biological control projects, 65 involved the release of only a single agent, 20 involved the release of two agents, and 23 involved the release of three or more agents. Twenty-five of the successful projects involved multiple agents (Table 2).

\subsection{Analysis}

For each project, we recorded the number of biological control agents that was released, the number which became established in at least one location, and the number of released agents that was involved in the success. The establishment rate was calculated as the number of agents established divided by the number of agents released in a biological control project, the success rate as the number of successful projects divided by the number of projects in each category (category $=$ projects with a specific number of agents released).

We analyzed the effect of the number of agents released in a biological control project on establishment rates using linear regression. The independent variable in this analysis was the number of agents used in a biological control project, and the dependent variable was the mean establishment rate. We also compared the establishment rates of agents in single versus multipleagent projects using two-tailed Mann-Whitney $\mathrm{U}$ tests.

We analyzed the effect of the number of agents released in a biological control project on success rates using logistic regression. Logistic regression is used when examining the relationship between a continuous independent variable and a binomial dependent variable (Trexler and Travis, 1993). In this case, the response variables are successful vs. unsuccessful. The computer software SYSTAT 8.0 for Windows (SPSS, 1998) was used to perform all statistical analyses. 
Table 1

Biological control projects against weeds

\begin{tabular}{|c|c|c|c|c|c|c|}
\hline S & Weed & $\begin{array}{l}\text { Country (State/ } \\
\text { Province) }\end{array}$ & $\mathrm{R}$ & $\mathrm{E}$ & I & Agents involved in success \\
\hline $\mathrm{Y}$ & Acacia saligna (Labillardière) Wendland & South Africa & 1 & 1 & 1 & Uromycladium tepperianum (Saccardo) McAlpine \\
\hline $\mathrm{N}$ & Acaena anserinifolia (Forster \& G. Forster) Druce & New Zealand & 1 & 0 & 0 & \\
\hline $\mathrm{N}$ & Acroptilon repens (Linnaeus) de Condolle & USA (WA) & 1 & 0 & 0 & \\
\hline $\mathrm{Y}$ & $\begin{array}{l}\text { Ageratina adenophora (Sprengel) R. King and H. } \\
\text { Robinson }\end{array}$ & USA (HI) & 2 & 1 & 1 & Procecidochares utilis Stone \\
\hline $\mathrm{Y}$ & Ageratina riparia (Regel) R. King and H. Robinson & USA (HI) & 4 & 3 & 3 & $\begin{array}{l}\text { Entyloma ageratinae Barreto and Evans, } \\
\text { Oidaematophorus beneficus Yano and Heppner, } \\
\text { Procecidochares alani Steyskal }\end{array}$ \\
\hline $\mathrm{Y}$ & Alternanthera philoxeroides (Martius) Grisebach & USA (FL) & 3 & 3 & 3 & $\begin{array}{l}\text { Agasicles hygrophila Selman and Vogt, Amynothrips } \\
\text { andersoni O'Neill, Arcola malloi (Pastrana) }\end{array}$ \\
\hline $\mathrm{N}$ & Ambrosia psilostachya de Candolle & Former USSR & 1 & 0 & 0 & \\
\hline $\mathrm{Y}$ & Baccharis halimifolia Linnaeus & Australia & 13 & 6 & 1 & Megacyllene mellyi (Chevrolat) \\
\hline $\mathrm{Y}$ & Carduus nutans group Linnaeus & Canada & 2 & 2 & 2 & $\begin{array}{l}\text { Rhinocyllus conicus (Frölich), Trichosirocalus } \\
\text { horridus (Panzer) }\end{array}$ \\
\hline $\mathrm{Y}$ & Centaurea diffusa Lamarck & Canada (BC) & 9 & 6 & 3 & $\begin{array}{l}\text { Agapeta zoegana Linnaeus, Sphenoptera jugoslavica } \\
\text { Obenberger, Urophora affinis (Frauenfeld) }\end{array}$ \\
\hline $\mathrm{Y}$ & Centaurea solstitialis Linnaeus & USA & 5 & 4 & 1 & Eustenopus villosus (Boheman) \\
\hline $\mathrm{N}$ & $\begin{array}{l}\text { Chromolaena odorata (Linnaeus) R. King and H. } \\
\text { Robinson }\end{array}$ & Nigeria & 2 & 0 & 0 & \\
\hline $\mathrm{N}$ & Cirsium arvense (Linnaeus) Scopoli & Canada (AB) & 4 & 2 & 0 & \\
\hline $\mathrm{Y}$ & Clidemia hirta (Linnaeus) D. Don & Fiji & 1 & 1 & 1 & Liothrips urichi Karny \\
\hline $\mathrm{N}$ & Convolvulus arvensis Linnaeus & USA & 2 & 1 & 0 & \\
\hline $\mathrm{Y}$ & Cordia curassavica (Jacquin) Roemer and Schultes & Mauritius & 3 & 2 & 2 & $\begin{array}{l}\text { Eurytoma attiva Burks, Metrogaleruca obscura } \\
\text { (Degeer) }\end{array}$ \\
\hline $\mathrm{N}$ & Cuscuta americana Linnaeus & Bahamas & 2 & 1 & 0 & \\
\hline $\mathrm{N}$ & Cyperus rotundus Linnaeus & USA (HI) & 2 & 2 & 0 & \\
\hline $\mathrm{N}$ & Cytiscus scoparius (Linnaeus) Link & USA (WA) & 2 & 2 & 0 & \\
\hline $\mathrm{Y}$ & Echium plantagineum Linnaeus & Australia & 2 & 2 & 1 & Mogulones larvatus Schultze \\
\hline $\mathrm{N}$ & Eichhornia crassipes (Martius) Solms-Laubach & Zambia & 3 & 1 & 0 & \\
\hline $\mathrm{N}$ & $\begin{array}{l}\text { Elephantopus mollis Humboldt, Bonpland and } \\
\text { Knuth }\end{array}$ & USA (HI) & 1 & 1 & 0 & \\
\hline $\mathrm{Y}$ & Emex australis Steinheil & USA (HI) & 3 & 1 & 1 & Perapion antiquum (Gyllenhal) \\
\hline $\mathrm{Y}$ & Euphorbia esula Linnaeus & Canada (SK) & 14 & 7 & 2 & $\begin{array}{l}\text { Aphthona cyparissiae (Koch), Aphthona nigriscutis } \\
\text { Foudras }\end{array}$ \\
\hline $\mathrm{N}$ & Galega officinalis Linnaeus & Chile & 1 & 1 & 0 & \\
\hline $\mathrm{N}$ & Halogeton glomeratus (M. Beib) C. Meyer & USA & 1 & 0 & 0 & \\
\hline $\mathrm{Y}$ & Harrisia martinii (Labouret) Britton and Rose & Australia & 4 & 3 & 1 & Hypogeococcus festerianus (Lizer y Trelles) \\
\hline $\mathrm{N}$ & Heliotropium europaeum Linnaeus & Australia & 1 & 0 & 0 & \\
\hline $\mathrm{Y}$ & Hydrilla verticillata (Linnaeus f) Royle & USA (FL) & 3 & 2 & 1 & Hydrellia pakistanae Deonier \\
\hline $\mathrm{Y}$ & Hypericum perforatum Linnaeus & Australia & 10 & 5 & 2 & $\begin{array}{l}\text { Chrysolina hyperici (Forster), Chrysolina } \\
\text { quadrigemina (Suffrian) }\end{array}$ \\
\hline $\mathrm{Y}$ & Lantana camara Linnaeus & USA (HI) & 25 & 15 & 8 & $\begin{array}{l}\text { Cremastobombycia lantanella Busck, Epinotia } \\
\text { lantana (Busck), Hypena laceratalis Walker, } \\
\text { Neogalea sunia (Guenée), Plagiohammus spinipennis } \\
\text { (Thomson), Salbia haemorrhoidalis Guenée, } \\
\text { Teleonemia scrupulosa } \text { Stål, Uroplata girardi Pic }\end{array}$ \\
\hline $\mathrm{N}$ & Linaria vulgaris Miller & Canada $(\mathrm{ON})$ & 1 & 1 & 0 & \\
\hline $\mathrm{Y}$ & Lythrum salicaria Linnaeus & USA & 2 & 2 & 2 & $\begin{array}{l}\text { Galerucella calmariensis (Linnaeus), Galerucella } \\
\text { pusilla (Duftschmidt) }\end{array}$ \\
\hline $\mathrm{Y}$ & Melastoma malabathricum Linnaeus & USA (HI) & 3 & 3 & 1 & Rhynchopalpus brunellus Hampson \\
\hline $\mathrm{N}$ & Mikania micrantha Knuth & $\begin{array}{l}\text { Solomon Is- } \\
\text { lands }\end{array}$ & 1 & 0 & 0 & \\
\hline $\mathrm{Y}$ & Opuntia imbricata (Haworth) de Candolle & Australia & 1 & 1 & 1 & Dactylopius tomentosus (Lamarck) \\
\hline $\mathrm{Y}$ & $\begin{array}{l}\text { Opuntia stricta (Haworth) var. stricta (Haworth) } \\
\text { Haworth }\end{array}$ & Australia & 11 & 5 & 2 & $\begin{array}{l}\text { Cactoblastis cactorum (Bergroth), Dactylopius } \\
\text { opuntiae (Cockerell) }\end{array}$ \\
\hline $\mathrm{Y}$ & Opuntia tomentosa Salm Dyck & Australia & 5 & 2 & 1 & Archlagocheirus funestus (Thomson) \\
\hline $\mathrm{Y}$ & Opuntia vulgaris Miller & India & 2 & 1 & 1 & Dactylopius ceylonicus (Green) \\
\hline $\mathrm{N}$ & Parkinsonia aculeata Linnaeus & Australia & 1 & 1 & 0 & \\
\hline $\mathrm{Y}$ & Parthenium hysterophorus Linnaeus & Australia & 6 & 5 & 4 & $\begin{array}{l}\text { Bucculatrix parthenica Bradley, Epiblema strenuana } \\
\text { (Walker), Listronotus setosipennis (Hustache), } \\
\text { Zygogramma bicolorata } \text { Pallister }\end{array}$ \\
\hline
\end{tabular}


Table 1 (continued)

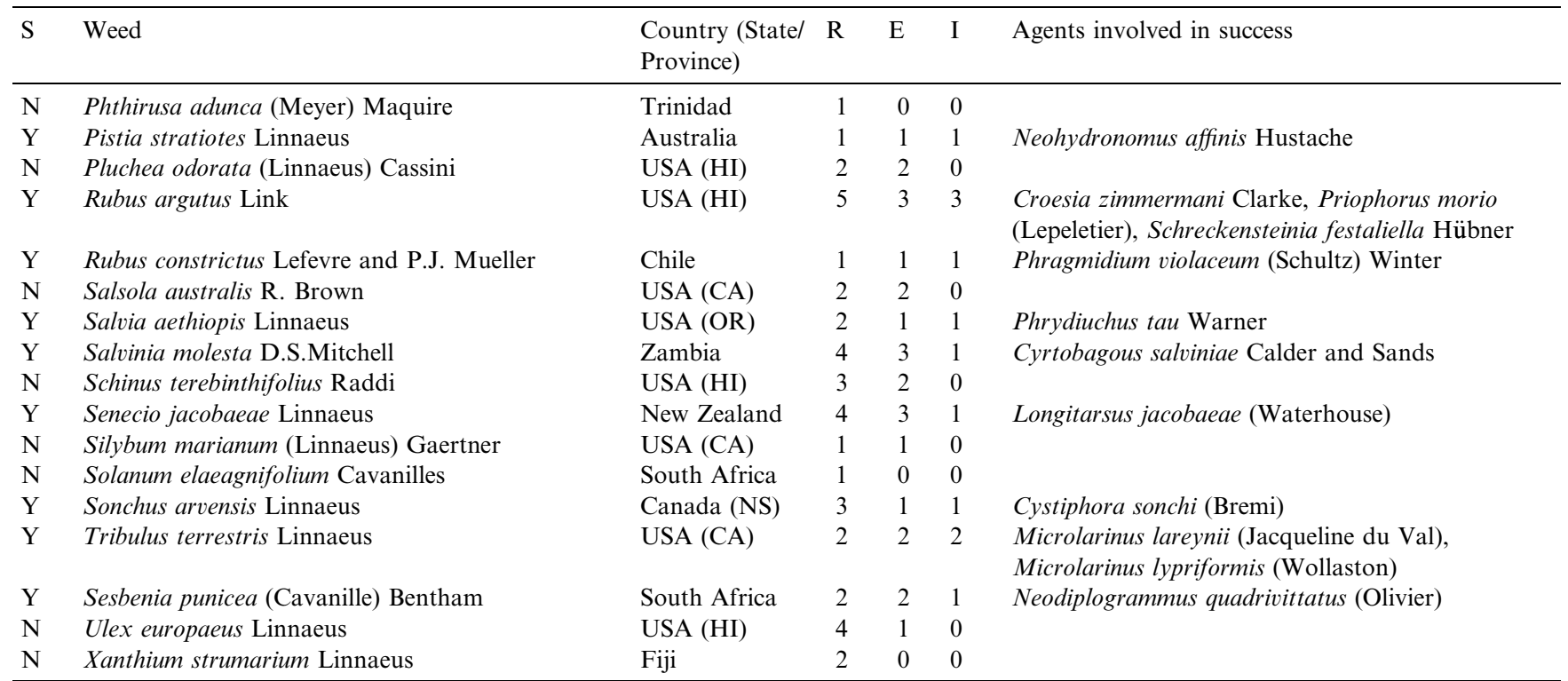

Note: Releases since 1990 not included in data set except when success was achieved with these agents; $\mathrm{S}$, the success of project $(\mathrm{Y}=$ yes, $\mathrm{N}=$ no); $\mathrm{R}$, the number of species released; E, the number of species established; I, the number of species involved in success.

To determine if the release of more control species also led to a higher number of species involved in the success, we compared the distribution of the number of agents released with that of the number of agents actually involved in success for all successful multipleagent projects in our data set.

\section{Results}

\subsection{Establishment rates}

The establishment rate of agents released was not associated with the number of agents in biological control projects against either weeds $\left(r^{2}=0.1\right.$, $P=0.3$; Fig. 1) or insects $\left(r^{2}=0.3, P=0.08\right.$; Fig. 2). However, the mean establishment rate of control agents in multiple-agent projects was significantly lower than in single-agent projects against insect pests $(U=1958, P=0.0001)$, but not weeds $(U=438.5$, $P=0.33$ ). On average, $75 \%$ of the agents in singleagent projects against insect pests established compared with only $47 \%$ in multiple-agent projects. For projects against weeds, the establishment rates were $53 \%$ in single-agent projects and $32 \%$ in multiple-agent projects.

\subsection{Success rates}

The success rate of biological control projects against weeds increased with the number of agents released $\left(r^{2}=0.23, P<0.0001 ;\right.$ Fig. 3). No relationship was found for insect pests $\left(r^{2}=0.001, P=0.33\right.$; Fig. 4).

\subsection{Number of agents involved in success}

For weeds, $75 \%$ of the 28 successful multiple-agent projects involved releases of two to five control agents and $25 \%$ involved six to 25 species (Fig. $5 \mathrm{a}$ ). In $54 \%$ of all projects involving the release of multiple agents, a single species was considered responsible for the success (Fig. 5b); in 39\%, two or three species. Four and eight agents, respectively, were considered to contribute to the success in two projects.

For insect biological control, $40 \%$ of the 25 successful multiple-agent projects involved the release of two control agents, $40 \%$ involved three to four agents and $20 \%$ involved five to 16 agents (Fig. 6a). In $56 \%$ of these projects, a single species was considered responsible for the success; in $44 \%$, two to four species (Fig. 6b).

\section{Discussion}

\subsection{Establishment rates}

The lower rate of establishment of biological control agents when multiple agents have been released has been the subject of intense discussion. Ehler and Hall (1982) found the same relationship in their study of control agents against insect pests and suggested that competitive exclusion may occur leading to the elimination of potentially superior control agents. They argued that future projects should avoid multiple-agent releases. Keller (1984) challenged this view and stated that the pest species is not a limiting resource and therefore, the agents were not likely to compete for hosts. Instead, 
Table 2

Biological control projects against insect pests

\begin{tabular}{|c|c|c|c|c|c|c|c|}
\hline S & Pest & $\begin{array}{l}\text { Country (State/ } \\
\text { Province) }\end{array}$ & $\mathrm{R}$ & $\mathrm{E}$ & I & Agents involved in success & Date \\
\hline $\mathrm{N}$ & Acyrthosiphon pisum (Harris) & Canada & 1 & 0 & 0 & & 1964-1967 \\
\hline $\mathrm{Y}$ & $\begin{array}{l}\text { Aleurocanthus spiniferus } \\
\text { (Quaintance) }\end{array}$ & Japan & 2 & 1 & 1 & Encarsia smithi (Silvestri) & 1925 \\
\hline $\mathrm{Y}$ & Aleurocanthus woglumi (Ashby) & Haiti & 1 & 1 & 1 & Eretmocerus serius (Silvestri) & 1930 \\
\hline $\mathrm{Y}$ & Aleurodicus cocois (Curtis) & Barbados & 2 & 1 & 1 & Encarsiella noyesi (Hayat) & 1950 \\
\hline $\mathrm{Y}$ & Aleurothrixus floccosus (Maskell) & Chile & 1 & 1 & 1 & Amitus spiniferus (Brethes) & 1967 \\
\hline $\mathrm{Y}$ & Anastrepha ludens (Loew) & Mexico & 11 & 3 & 1 & Aceratoneuromyia indica (Silvestri) & $1954-1955$ \\
\hline $\mathrm{N}$ & Anastrepha striata (Schiner) & Trinidad & 1 & 0 & 0 & & $1973-1975$ \\
\hline $\mathrm{N}$ & Anthonomus grandis (Boheman) & USA & 1 & 0 & 0 & & 1966 \\
\hline $\mathrm{Y}$ & Antonina graminis (Maskell) & USA & 1 & 1 & 1 & Neodusmetia sangwani (Subba Rao) & $1958-1959$ \\
\hline $\mathrm{Y}$ & Aonidiella aurantii (Maskell) & South Africa & 1 & 1 & 1 & Rhyzobius lophanthae (Blaisdell) & 1900 \\
\hline $\mathrm{N}$ & Aphids & New Zealand & 4 & 0 & 0 & & $1968-1973$ \\
\hline $\mathrm{N}$ & Aphis craccivora (Koch) & India & 1 & 0 & 0 & & 1966 \\
\hline $\mathrm{Y}$ & Aphis nerii (Fonscolombe) & USA (Hawaii) & 1 & 1 & 1 & Lysiphlebus testaceipes (Cresson) & 1965 \\
\hline $\mathrm{Y}$ & Argyroploce schistaceana (Snellen) & $\begin{array}{l}\text { Marianas (Sai- } \\
\text { pan) }\end{array}$ & 1 & 1 & 1 & Trichogramma chilonis (Ishii) & 1935 \\
\hline $\mathrm{N}$ & Aspidiotus destructor (Signoret) & $\begin{array}{l}\text { Marianas (Pon- } \\
\text { ape) }\end{array}$ & 1 & 1 & 0 & & 1987 \\
\hline $\mathrm{Y}$ & Asterolecanium variolosum (Ratzeburg) & Chile & 1 & 1 & 1 & Habrolepis dalmani (Westwood) & 1928 \\
\hline $\mathrm{Y}$ & Bactrocera cucurbitae (Coquillett) & USA (Hawaii) & 1 & 1 & 1 & Psyttalia fletcheri (Silvestri) & 1916 \\
\hline $\mathrm{Y}$ & Bactrocera dorsalis (Hendel) & USA (Hawaii) & 16 & 5 & 1 & Fopius arisanus (Sonan) & $1947-1953$ \\
\hline $\mathrm{Y}$ & Brontispa longissima (Gestro) & Indonesia & 1 & 1 & 1 & Tetrastichus brontispae (Ferriere) & $1932-1933$ \\
\hline $\mathrm{N}$ & Carpophilus hemipterus (Linnaeus) & USA (Hawaii) & 2 & 1 & 0 & & $1976-1977$ \\
\hline $\mathrm{Y}$ & Ceratitis capitata (Wiedemann) & Spain & 1 & 1 & 1 & Tetrastichus giffardianus (Silvestri) & 1959 \\
\hline $\mathrm{Y}$ & Ceroplastes rubens (Maskell) & Australia & 1 & 1 & 1 & $\begin{array}{l}\text { Anicetus beneficus (Ishii and } \\
\text { Yasumatsu) }\end{array}$ & 1977 \\
\hline $\mathrm{N}$ & Chilo partellus (Swinhoe) & Comoro Islands & 3 & 0 & 0 & & $1981-1983$ \\
\hline $\mathrm{N}$ & Chilo sacchariphagus (Bojer) & Madagascar & 1 & 0 & 0 & & 1962 \\
\hline $\mathrm{N}$ & Chilo terrenellus Pagenstecher & $\begin{array}{l}\text { Papua New } \\
\text { Guinea }\end{array}$ & 1 & 0 & 0 & & 1981 \\
\hline $\mathrm{N}$ & Choristoneura fumiferana (Clemens) & Canada & 1 & 0 & 0 & & 1955 \\
\hline $\mathrm{N}$ & Clemora smithi (Arrow) & Mauritius & 1 & 1 & 0 & & 1913 \\
\hline $\mathrm{Y}$ & Cnidocampa flavescens (Walker) & USA & 1 & 1 & 1 & $\begin{array}{l}\text { Chaetexorista javana (Brauer and } \\
\text { Bergenstamm) }\end{array}$ & 1930 \\
\hline $\mathrm{N}$ & Coccus hesperidum (Linnaeus) & USA & 1 & 1 & 0 & & 1938 \\
\hline $\mathrm{N}$ & Coccus pseudomagnoliarum (Kuwana) & USA & 2 & 0 & 0 & & 1953 \\
\hline $\mathrm{Y}$ & Cockroaches & USA (Hawaii) & 1 & 1 & 1 & Dolichurus stantoni (Ashmead) & 1917 \\
\hline $\mathrm{Y}$ & Cosmopolites sordidus (Germar) & Fiji & 1 & 1 & 1 & Plaesius laevigatus (Marseul) & 1918 \\
\hline $\mathrm{N}$ & Cydia molesta (Busck) & USA & 1 & 1 & 0 & & 1927 \\
\hline $\mathrm{N}$ & Dialeurodes citri (Ashmead) & Greece & 1 & 1 & 0 & & 1976 \\
\hline $\mathrm{Y}$ & Diaphorina citri (Kuwayama) & Reunion & 1 & 1 & 1 & Tamarixia radiata (Waterston) & 1978 \\
\hline $\mathrm{N}$ & Diatraea centrella (Moschler) & Guyana & 2 & 0 & 0 & & $1960-1961$ \\
\hline $\mathrm{Y}$ & Diatraea saccharalis (Fabricius) & St. Kits & 1 & 1 & 1 & Lixophaga diatraeae (Townsend) & 1932 \\
\hline $\mathrm{N}$ & Diprion frutetorum (Fabricius) & Canada & 2 & 0 & 0 & & 1942 \\
\hline $\mathrm{Y}$ & Dryocasmus kuriphilus (Yasumatsu) & Japan & 1 & 1 & 1 & Torymus sinensis (Kamijo) & 1982 \\
\hline $\mathrm{N}$ & Dysmicoccus boninsis (Kuwana) & Bahamas & 4 & 1 & 0 & & 1968 \\
\hline $\mathrm{N}$ & Dysmicoccus brevipes (Cockerell) & Puerto Rico & 2 & 1 & 0 & & $1937-1938$ \\
\hline $\mathrm{N}$ & Earias vitella (Fabricius) & Fiji & 1 & 0 & 0 & & 1971 \\
\hline $\mathrm{Y}$ & Edwardsiana froggatti (Baker) & Tasmania & 1 & 1 & 1 & Anagrus armatus nigriventris Burks & 1935 \\
\hline $\mathrm{Y}$ & Eriococcus coriaceus (Maskell) & New Zealand & 2 & 2 & 1 & Rhyzobius ventralis (Erichson) & 1905 \\
\hline $\mathrm{Y}$ & Erionota thrax (Linnaeus) & Mauritius & 4 & 2 & 2 & $\begin{array}{l}\text { Ooencyrtus erionotae (Ferriere), } \\
\text { Apanteles erionotae (Wilkinson) }\end{array}$ & 1971-1972 \\
\hline $\mathrm{Y}$ & Eriosoma lanigerum (Haussmann) & Argentina & 1 & 1 & 1 & Aphelinus mali (Haldeman) & 1920 \\
\hline $\mathrm{Y}$ & Gonipterus scutellatus (Gyllenhal) & New Zealand & 1 & 1 & 1 & Anaphes nitens (Girault) & $1927-1930$ \\
\hline $\mathrm{Y}$ & Gossyparia spuria (Modeer) & USA & 1 & 1 & 1 & Coccophagus insidiator (Dalman) & 1939 \\
\hline $\mathrm{Y}$ & Gynaikothrips ficorum (Marchal) & Bermuda & 1 & 1 & 1 & Montandoniola moraguesi (Puton) & 1973 \\
\hline $\mathrm{N}$ & Helicoverpa armigera (Hubner) & India & 2 & 0 & 0 & & 1968 \\
\hline $\mathrm{N}$ & Helicoverpa zea (Boddie) & USA (Hawaii) & 1 & 1 & 0 & & 1951 \\
\hline $\mathrm{N}$ & Heliothis virescens (Fabricius) & Barbados & 2 & 0 & 0 & & $1972-1976$ \\
\hline $\mathrm{N}$ & Hemiberlesia lataniae (Signoret) & New Zealand & 3 & 0 & 0 & & $1987-1990$ \\
\hline $\mathrm{N}$ & Heteronychus arator (Fabricius) & New Zealand & 1 & 0 & 0 & & 1967-1969 \\
\hline $\mathrm{Y}$ & Homona coffearia (Nietner) & Sri Lanka & 1 & 1 & 1 & Macrocentrus homonae (Nixon) & $1935-1936$ \\
\hline
\end{tabular}


Table 2 (continued)

\begin{tabular}{|c|c|c|c|c|c|c|c|}
\hline $\mathrm{S}$ & Pest & $\begin{array}{l}\text { Country (State/ } \\
\text { Province) }\end{array}$ & $\mathrm{R}$ & $\mathrm{E}$ & I & Agents involved in success & Date \\
\hline Y & Houseflies and Stableflies & USA (Hawaii) & 3 & 3 & 3 & $\begin{array}{l}\text { Pachycrepoideus vindemiae } \\
\text { (Rondani), Spalangia cameroni } \\
\text { (Perkins), Spalangia endius } \\
\text { philippensis (Walker) }\end{array}$ & $1914-1920$ \\
\hline Y & Hypothenemus hampei (Ferrari) & Brazil & 1 & 1 & 1 & Prorops nasuta (Waterston) & 1929 \\
\hline Y & Icerya aegyptiaca (Douglas) & Marianas & 1 & 1 & 1 & Rodolia pumila Weise & 1928 \\
\hline Y & $\begin{array}{l}\text { Icerya montserratensis (Riley and } \\
\text { Howard) }\end{array}$ & Ecuador & 1 & 1 & 1 & Rodolia cardinalis (Mulsant) & 1942 \\
\hline $\mathrm{N}$ & Lepidiota pruinosa Wiedemann & Philippines & 2 & 0 & 0 & & 1931 \\
\hline $\mathrm{N}$ & Listronotus bonariensis (Kuschel) & New Zealand & 2 & 0 & 0 & & $1966-1967$ \\
\hline $\mathrm{N}$ & Lygus spp. Hahn & USA & 1 & 0 & 0 & & 1980 \\
\hline $\mathrm{N}$ & Lymantria dispar (Linnaeus) & Portugal & 1 & 1 & 0 & & 1932 \\
\hline $\mathrm{Y}$ & Maruca testulalis Geyer & Mauritius & 7 & 2 & 2 & $\begin{array}{l}\text { Eiphosoma dentator (Fabricius), } \\
\text { Bracon cajani Muesebeck }\end{array}$ & $1953-1956$ \\
\hline Y & Mealybugs & Portugal & 1 & 1 & 1 & Cryptolaemus montrouzieri Mulsant & 1929 \\
\hline Y & Melanaphis sacchari (Zehntner) & USA (Hawaii) & 3 & 3 & 3 & $\begin{array}{l}\text { Coelophora inaequalis (Fabricius), } \\
\text { Platyomus lividagaster Mulsant, } \\
\text { Scymnus notescens Blackburn }\end{array}$ & 1894 \\
\hline $\mathrm{N}$ & Musca domestica Linnaeus & USA (Hawaii) & 2 & 1 & 0 & & 1967 \\
\hline $\mathrm{N}$ & Nezara viridula (Linnaeus) & USA (Hawaii) & 1 & 0 & 0 & & 1967 \\
\hline Y & Ophiomyia phaseoli (Tryon) & USA (Hawaii) & 2 & 2 & 2 & $\begin{array}{l}\text { Opius phaseoli Fischer, Opius } \\
\text { importatus Fischer }\end{array}$ & 1969 \\
\hline $\mathrm{N}$ & Opisina arenosella Walker & Sri Lanka & 4 & 0 & 0 & & 1959-1977 \\
\hline Y & Orthezia insignis Browne & USA (Hawaii) & 1 & 1 & 1 & Hyperaspis pantherina Fursch & 1907 \\
\hline $\mathrm{N}$ & Oryctes monoceros Olivier & Seychelles & 1 & 0 & 0 & & 1960 \\
\hline $\mathrm{N}$ & Oryctes rhinoceros Linnaeus & Mauritius & 8 & 4 & 0 & & $1962-1967$ \\
\hline $\mathrm{Y}$ & Papilio polytes Linnaeus & Marianas & 1 & 1 & 1 & Pteromalus luzonensis Gahan & $1973-1974$ \\
\hline $\mathrm{N}$ & Paramyelois transitella (Walker) & USA & 1 & 0 & 0 & & 1973 \\
\hline $\mathrm{Y}$ & Parlatoria oleae (Colvee) & USA & 2 & 2 & 1 & Aphytis maculicornis (Masi) & 1951 \\
\hline Y & Perkinsiella saccharicida Kirkaldy & USA (Hawaii) & 5 & 4 & 1 & Anagrus optabilis (Perkins) & 1904-1905 \\
\hline $\mathrm{N}$ & Phthorimaea operculella (Zeller) & India & 6 & 3 & 0 & & 1964 \\
\hline $\mathrm{Y}$ & Pinnaspis buxi (Baschie) & USA (Hawaii) & 1 & 1 & 1 & Telsimia nitida Chapin & 1936 \\
\hline Y & Planococcus kenyae (LePelly) & Kenya & 5 & 5 & 1 & Anagyrus kivuensis Compere & 1938 \\
\hline $\mathrm{N}$ & Plutella xylostella (Linnaeus) & Cook Islands & 3 & 1 & 0 & & $1974-1975$ \\
\hline $\mathrm{N}$ & Pristiphora erichsonii Hartig & USA & 1 & 1 & 0 & & 1911 \\
\hline $\mathrm{Y}$ & Promecotheca opacicollis Gestro & Vanuatu & 1 & 1 & 1 & Pediobius parvulus (Ferrier) & 1937 \\
\hline $\mathrm{Y}$ & $\begin{array}{l}\text { Pseudaulacaspis pentagona } \\
\text { (Targioni-Tozzetti) }\end{array}$ & Italy & 1 & 1 & 1 & Encarsia berlesei (Howard) & 1906 \\
\hline Y & Pseudococcus calceolariae (Maskell) & USA & 6 & 2 & 2 & $\begin{array}{l}\text { Coccophagus gurneyi Compere, } \\
\text { Arhopoideus pretiosus (Timberlake) }\end{array}$ & $1927-1928$ \\
\hline $\mathrm{Y}$ & Pseudococcus citriculus Green & Israel & 1 & 1 & 1 & Clausenia purpurea Ishii & 1940 \\
\hline $\mathrm{Y}$ & Pseudococcus comstocki (Kuwana) & USSR & 2 & 1 & 1 & Coccophagus gurneyi Compere & 1939-1942 \\
\hline Y & $\begin{array}{l}\text { Pseudococcus longispinus } \\
\text { (Targioni-Tozzetti) }\end{array}$ & USA & 2 & 2 & 2 & $\begin{array}{l}\text { Arhopoideus peregrinus (Compere), } \\
\text { Tetracnemoidea sydneyensis } \\
\text { (Timberlake) }\end{array}$ & 1933-1934 \\
\hline $\mathrm{Y}$ & Quadraspidiotus perniciosus (Comstock) & Italy & 1 & 1 & 1 & Encarsia perniciosi (Tower) & 1947 \\
\hline $\mathrm{Y}$ & Rhabdoscelus obscurus (Boisduval) & Australia & 1 & 1 & 1 & Lixophaga sphenophori (Villeneuve) & 1910 \\
\hline $\mathrm{Y}$ & Saccharicoccus sacchari (Cockerell) & Barbados & 1 & 1 & 1 & Anagyrus saccharicola Timberlake & 1870 \\
\hline $\mathrm{Y}$ & Saissetia oleae (Olivier) & USA & 1 & 1 & 1 & Rhyzobius ventralis (Erichson) & $1888-1892$ \\
\hline Y & Scale Insects & Seychelles & 5 & 4 & 4 & $\begin{array}{l}\text { Chilocorus distigma (Klug), } \\
\text { Chilocorus nigritus (Fabricius), } \\
\text { Exochomus flavipes (Thunberg), } \\
\text { Exochomus ventralis Gerstaecker }\end{array}$ & 1936 \\
\hline $\mathrm{Y}$ & Sesamia calamistis Hampson & Mauritius & 1 & 1 & 1 & Cotesia sesamiae (Cameron) & $1951-1952$ \\
\hline Y & Sesamia nonagrioides Lefebvre & $\begin{array}{l}\text { Cape Verde Is- } \\
\text { lands }\end{array}$ & 2 & 2 & 1 & Pediobius furvus Gahan & 1984-1987 \\
\hline Y & Sexava spp. Walker & $\begin{array}{l}\text { Papua New } \\
\text { Guinea }\end{array}$ & 2 & 1 & 1 & Leefmansia bicolor Waterston & 1929-1933 \\
\hline Y & Siphanta acuta (Walker) & USA (Hawaii) & 3 & 1 & 1 & Aphanomerus pusillus Perkins & 1904 \\
\hline $\mathrm{Y}$ & Spodoptera exempta (Walker) & USA (Hawaii) & 4 & 4 & 3 & $\begin{array}{l}\text { Glyptapanteles militaris (Walsh), } \\
\text { Euplectrus platyhypenae Howard, } \\
\text { Archytas cirphis Curran }\end{array}$ & 1923-1924 \\
\hline
\end{tabular}


Table 2 (continued)

\begin{tabular}{|c|c|c|c|c|c|c|c|}
\hline $\mathrm{S}$ & Pest & $\begin{array}{l}\text { Country (State/ } \\
\text { Province) }\end{array}$ & $\mathrm{R}$ & $\mathrm{E}$ & I & Agents involved in success & Date \\
\hline $\mathrm{Y}$ & Spodoptera litura (Fabricius) & Marianas & 1 & 1 & 1 & Telenomus nawaii Ashmead & 1936 \\
\hline $\mathrm{N}$ & Stomoxys calcitrans (Linnaeus) & Mauritius & 1 & 0 & 0 & & 1953 \\
\hline $\mathrm{Y}$ & Therioaphis trifolii Monell & USA & 10 & 3 & 3 & $\begin{array}{l}\text { Aphelinus asychis Walker, } \\
\text { Praon exsoletum } \text { (Nees), } \\
\text { Trioxys complanatus } \text { Quilis }\end{array}$ & $1955-1957$ \\
\hline $\mathrm{N}$ & Trichoplusia ni (Hubner) & Trinidad & 1 & 0 & 0 & & 1976 \\
\hline $\mathrm{Y}$ & Trioza erytreae (Del Guercio) & Reunion & 1 & 1 & 1 & Tamarixia dryi Waterston & 1974 \\
\hline $\mathrm{Y}$ & Unaspis yanonensis (Kuwana) & Japan & 2 & 2 & 2 & $\begin{array}{l}\text { Aphytis yanonensis Debach and } \\
\text { Rosen, Coccobius fulvus } \\
\text { (Compere and Annecke) }\end{array}$ & 1980 \\
\hline
\end{tabular}

Note: Releases after 1990 not included in data set except when success was achieved with these agents; $\mathrm{S}$, the success of project ( $\mathrm{Y}=\mathrm{yes}, \mathrm{N}=$ no); $\mathrm{R}$, the number of species released; $\mathrm{E}$, the number of species established; I, the number of species involved in success.

he proposed other hypotheses that are consistent with the evidence, such as bias in the data, the sequence of release of the control agents and learning effects that

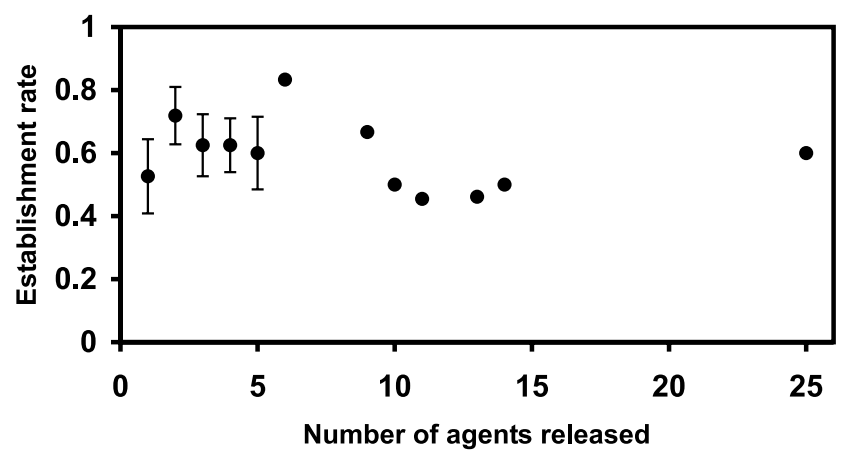

Fig. 1. Mean establishment rates $( \pm$ SEM) of biological control agents released against weeds, given the number of agents released in a biological control project. Error bars could not be produced for projects with a sample size of 1 . There is no significant relationship $\left(r^{2}=0.1\right.$, $P=0.3)$.

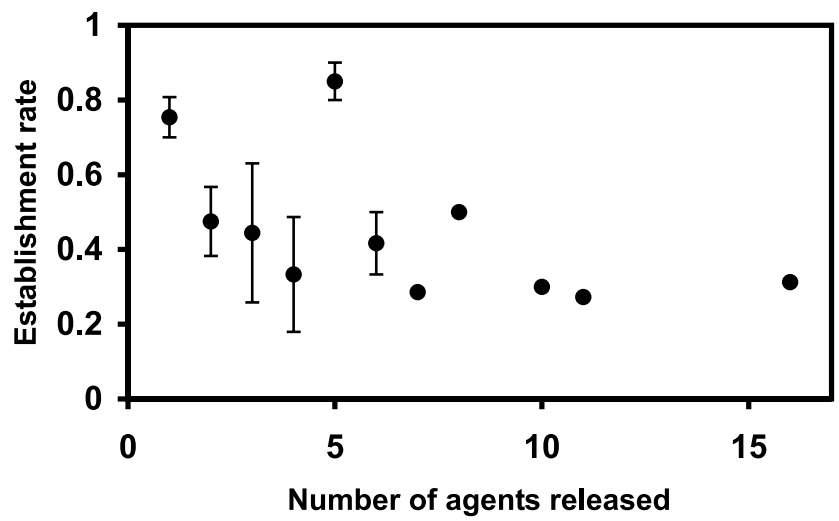

Fig. 2. Mean establishment rates $( \pm$ SEM) of biological control agents released against insect pests, given the number of agents released in a biological control project. Error bars could not be produced for projects with a sample size of 1 . The relationship is not significant $\left(r^{2}=0.3, P=0.08\right)$. influence later starting projects against a certain pest. An alternative explanation is that control agents are released until one or more establish. Thus, the number

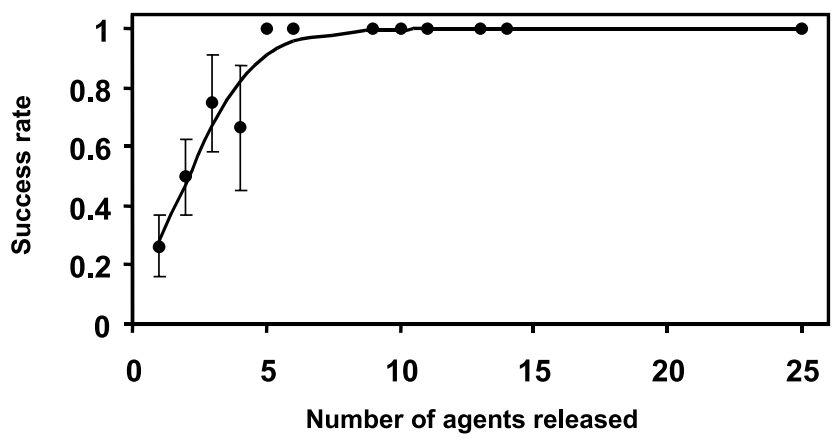

Fig. 3. The probability of success for biological control projects against weeds as a function of the number of agents released. The curve shows the predictions of the logistic regression model $\left(r^{2}=0.23, P<0.001\right)$ while the points show the average success rates $( \pm$ SEM) for projects in the data set. Error bars could not be produced for projects with a sample size of 1 .

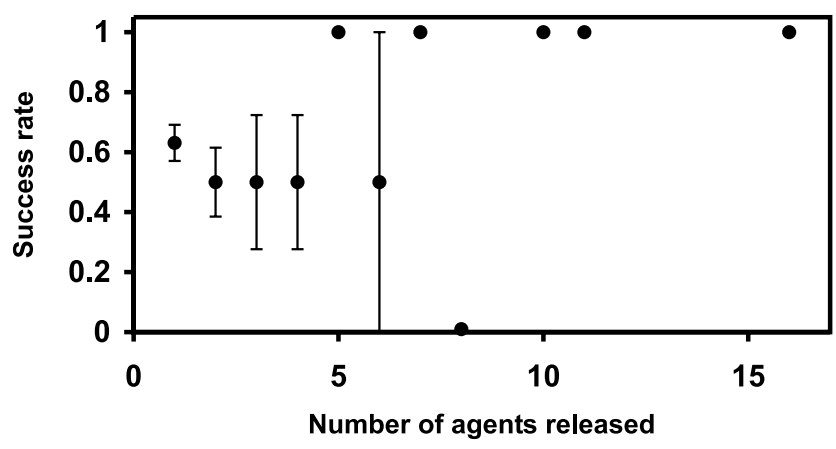

Fig. 4. The average success rate $( \pm$ SEM) of biological control projects against insect pests, given the number of agents released. The logistic regression model was not significant $\left(r^{2}=0.001, P=0.33\right)$. Error bars could not be produced for projects with a sample size of 1 . 

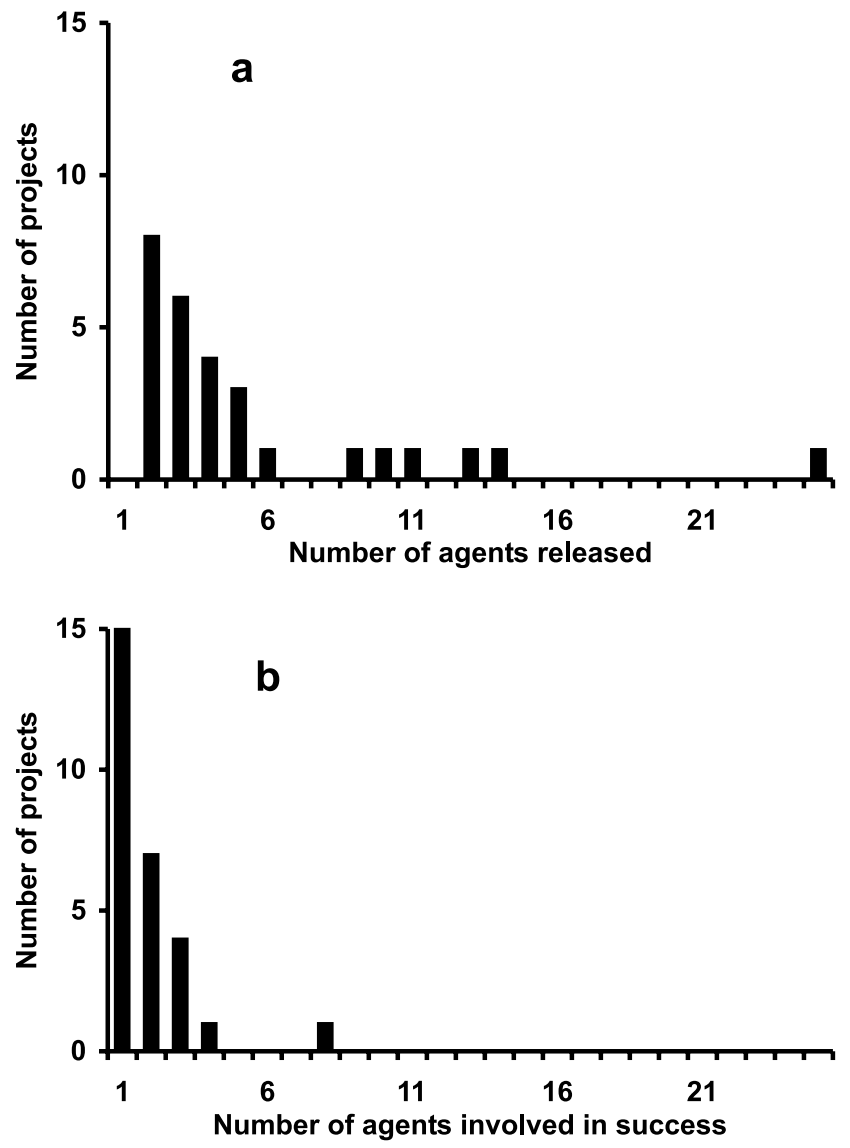

Fig. 5. The number of agents released (a) or involved in success (b) for successful, multiple-agent projects against weeds.

of agents may be dependent on the establishment of previously introduced agents. Given that our data and the data used by Ehler and Hall (1982) are both observational, the problem calls for experimental studies to examine the role of competition in producing the observed establishment rates. However, until further evidence has been gathered, competition remains a viable hypothesis.

In contrast, the establishment rates of agents in projects against weeds did not vary in single vs. multiple-agent projects. Three factors could contribute to the observed difference between insect and weed biological control. The first is the possibility of intraguild predation where two biological control agents enter a predator-prey relationship. Rosenheim et al. (1995) found that intraguild predation occurs frequently among biological control agents against insect pests but is rare among biological control agents against weeds. Second, plants are not often killed by herbivores and offer a range of host niches. Therefore, a single host may support multiple agents. Third, control agents against insects may interfere with each other more than control agents released against weeds due to the continued presence of host individuals that are
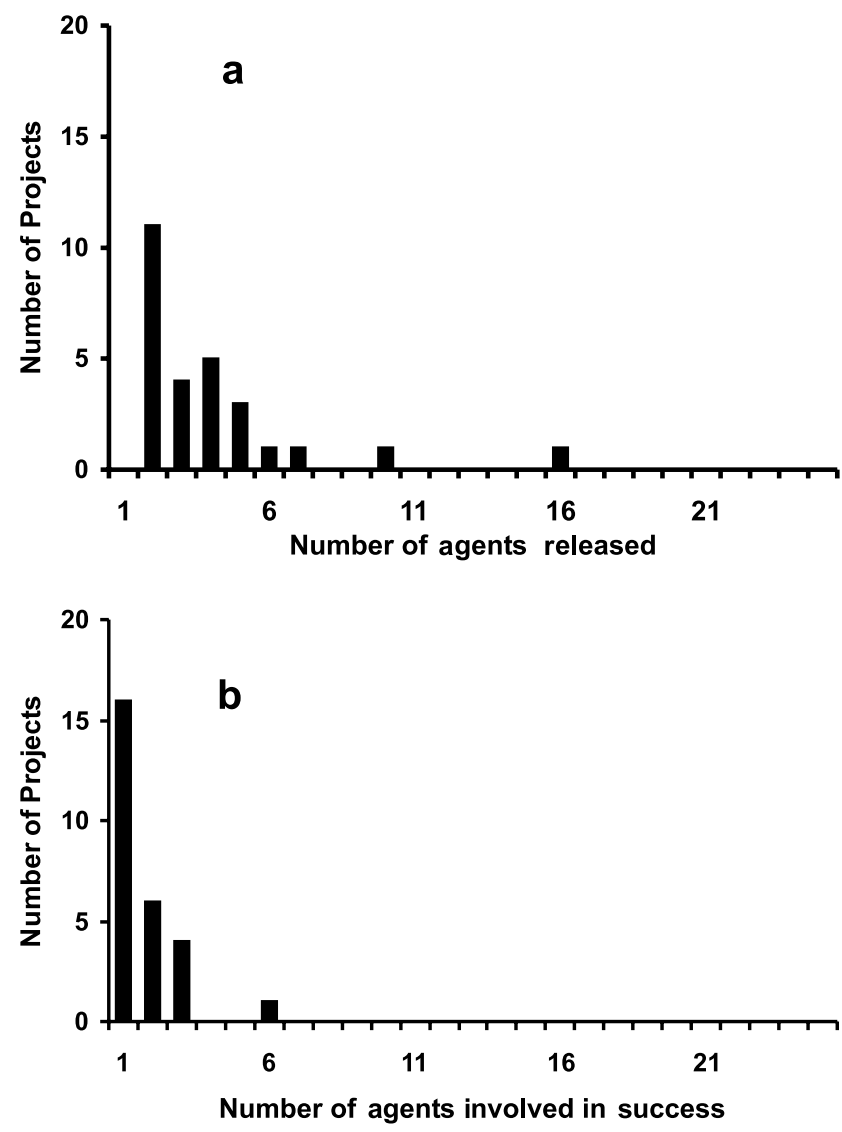

Fig. 6. The number of agents released (a) or involved in success (b) for successful, multiple-agent projects against insect pests.

already infested by an agent (parasitoid) but are apparently still available for other species. More than one attack on the same host individual could result in the reproductive failure, or a reduction in fitness, of one or all of the parasitoids involved (De Moraes et al., 1999; Leveque et al., 1993; Reitz, 1996). In some cases, the parasitoid species that is most negatively influenced by competition is the most effective control agent when alone (Leveque et al., 1993). Therefore, careful consideration of competition between control agents against insect pests is necessary prior to their release.

Competition between biological control agents against weeds may not be as common as between agents against insect pests because once a plant part is consumed it is unavailable for subsequent attack. However, a recent review of the literature found evidence for competitive interactions among phytophagous insect biological control agents in $91 \%$ of the 45 examined experimental studies between two species (Denno et al., 1995). Competitive interactions between biological control agents against weeds may not be a function of the number of agents released, but competition does occurs. 
One example of competition between biological control agents against weeds is in the ongoing effort to control purple loosestrife, Lythrum salicaria (L.), in North America (Table 1). Four control agents were released. Two of these agents, Galerucella calmariensis (L.) and Galerucella pusilla (Duftschmidt), feed primarily on the foliage. After heavy defoliation, the flowering of purple loosestrife is suppressed, resulting in a food shortage for another control agent, Nanophyes marmoratus Goeze, a flower-feeder (Julien and Griffiths, 1998). As with biological control agents against insect pests, control agents against weeds should be selected carefully so as to minimize competitive interactions and maximize impact on plant density. Unnecessary agents should not be introduced.

\subsection{Success rates}

Our survey shows that in multiple-agent projects, success was not higher than in single-agent projects against insect pests. This result may be due to the lower establishment rate of agents in multiple-agent projects. A study supporting this view (Ferguson and Stiling, 1996) found that interference of predators and parasitoids of aphids reduced mortality of the host. Alternatively, the following interpretation is possible: introductions of control agents are nonrandom events, in that practitioners are likely to release the most promising agents first. If no success is achieved, the subsequent release of less suitable agents is even less likely to result in success. Regardless of the underlying cause, the observed result could indicate that introducing more agents is not useful, since it does not necessarily enhance success.

In the biological control of weeds, success rates increased significantly with the number of agents released. However, it should be noted that only $23 \%$ of the variation in success rate was explained by the number of agents released (Fig. 3). This indicates that the number of agents released is not a reliable predictor of success rate and that other factors, such as climatic match, or interactions with localized natural enemies, may play an influential role in the success of biological control projects. Given that practitioners have control over how many agents are released, it is worth examining the consequences of releasing more species. A higher success rate of biological control projects with multiple agents is predicted by two models: in the lottery model (Myers, 1985), the release of more agents increases the chance of success because it is more likely that the "right" agent is released; in the cumulative stress model (Harris, 1985; Myers, 1985), multiple biological control agents may be needed to generate sufficient damage to the weed. To differentiate between the lottery model and the cumulative stress model, it is necessary to examine how many of the released agents were involved in the success. A unique prediction of the lottery model is that one agent only is usually responsible for the success, while several agents are expected to be involved in the success in the cumulative stress model.

\subsection{Number of agents involved in success}

In our analysis, a single agent was sufficient to reduce both insect and weed pests in more than $50 \%$ of multiple-agent projects in which several species were released. Myers (1985) and Myers et al. (1989) found that in $81 \%$ (21 of 26) of successful control projects against weeds and in 68\% (34 of 50) of successful control projects against insect pests, a single species was considered to be responsible for the success. Whereas these figures are higher than in the current study, the trend in the data is the same. These results are consistent with the hypothesis that, for a majority of biological control projects, release of the "right" control agent (lottery model), rather than a complex of species (cumulative stress model), is more likely to achieve success and that only some predators are able to reduce their prey (Myers, 1985). In over $40 \%$ of the projects, however, two, three, or even more species were considered to be involved in reducing the pest. This indicates that for some pests a complex of control agents may be required. More than one agent may be needed if the pest species is living in several different environments, and one agent is only effective in a part of the pest species' range. However, projects in which success is attributed to more than one agent may be indicative of experimenter bias rather than of a necessity to use multiple agents. Julien et al. (1984) suggested that while the failure of an agent to control a weed is obvious when it is released alone, it may be viewed as contributing to success when released with a complex of species that provide successful control. In the reported successful biological control of diffuse knapweed, Centaurea diffusa Lamarck (Table 1), for instance, three agents are considered to be involved even though quantitative plant density data are either lacking or indicate little impact (Myers and Risley, 2000). Thus, the number of projects in which the success is attributed to several agents may be too high. While there may be instances when multiple agents are needed for successful control, this is not the case for the majority of biological control projects.

Although we acknowledge the difficulty of finding the "right" species, the introduction of multiple agents should be conducted with caution. The risks from introductions are twofold: (1) negative impacts on the control projects themselves and (2) direct and indirect effects on nontarget species.

Negative impacts on the biological control projects themselves could result from competitive interactions or intraguild predation amongst control agents 
(Rosenheim et al., 1995). Ideally, any additional agent should be released only if a previously introduced species does not control the pest. When several control agents are released simultaneously, they should be introduced separately in infestations sufficiently isolated from each other to avoid negative interactions and to allow for a scientifically sound evaluation of the efficacy of the individual agents. Following Julien et al. (1984), we consider careful evaluations of past projects essential for progress in the field of biological control.

The direct risk of attacks on nontarget species has certainly decreased since rigorous host specificity testing for biological control agents of weeds is required. These tests have also advanced the knowledge about the control agents and may have increased their chance of establishment. Given the growing concerns about the safety of natural enemy introductions in general, such tests will likely be required for control agents against insect pests in the future.

The current interest in indirect, negative effects on native species by introduced control agents has stimulated discussion about the prevalence of such interactions, although hardly any evidence exists. We only know of the study of Callaway et al. (1999) who found that the performance of a native grass in the presence of an invasive weed was reduced when the introduced control agent attacked the weed. The authors hypothesized that the attacked plants produced chemicals with negative effects on the neighboring plants. However, the biomass of the weed was not reduced by the attacks. The lack of further evidence for indirect, negative effects is possibly due to the difficulty of assessing such effects (Fowler et al., 2000) and the absence of research (Hopper, 1998). Taking into account, however, that each introduced control agent adds to the complexity of a natural system and may entail negative effects, the risks from introductions should be carefully balanced against the potential benefits.

Bearing in mind that one agent alone is sufficient to control most pests and considering the potential risks for the projects themselves and the native fauna and flora, we recommend restraint in the introduction of multiple agents for biological control.

\section{Acknowledgments}

We thank three anonymous reviewers for helpful comments that improved the paper. Financial support was provided by scholarships from The National Science and Engineering Research Council (NSERC), The Entomological Society of Canada and The Entomological Society of British Columbia to L. Frid and by an NSERC operating grant to J. Myers.

\section{References}

Bellows, T.S., 2001. Restoring population balance through natural enemy introductions. Biol. Control 21, 199-205.

Callaway, R.M., DeLuca, T.H., Belliveau, W.M., 1999. Biologicalcontrol herbivores may increase competitive ability of the noxious weed Centaurea maculosa. Ecology 80, 1196-1201.

Caltagirone, L.E., 1981. Landmark examples in classical biological control. Annu. Rev. Entomol. 26, 213-232.

Cory, J.S., Myers, J.H., 2000. Direct and indirect ecological effects of biological control. TREE 15, 137-139.

De Moraes, C.M., Cortesero, A.M., Stapel, J.O., Lewis, W.J., 1999. Intrinsic and extrinsic competitive interactions between two larval parasitoids of Heliothis virescens. Ecol. Entomol. 24, 402-410.

Denno, R.F, McClure, M.S., Ott, J.R., 1995. Interspecific interactions in phytophagous insects: competition reexamined and resurrected. Annu. Rev. Entomol. 40, 297-331.

Ehler, L.E., Hall, R.W., 1982. Evidence for competitive exclusion of introduced natural enemies in biological control. Environ. Entomol. 11, 1-4.

Ferguson, K.I., Stiling, P., 1996. Non-additive effects of multiple natural enemies on aphid populations. Oecologia 108, 375-379.

Fowler, S.V., Syrett, P., Hill, R.L., 2000. Success and safety in the biological control of environmental weeds in New Zealand. Aust. Ecol. 25, 553-562.

Greathead, D.J., Greathead, A.H., 1992. Biological control of insect pests by insect parasitoids and predators: the BIOCAT database. Biocontrol News Inform. 13, 61-68.

Harris, P., 1985. Biological control of weeds: bureaucrats, botanists, beekeepers, and other bottlenecks. In: Delfosse, E.S. (Ed.), Proceedings of the 6th International Symposium on the Biological Control of Weeds, Agriculture Canada. Canadian Govt. Printing Office, Ottawa, pp. 3-12.

Hawkins, B.A., 1993. Parasitoid richness, host mortality, and biological control. Am. Nat. 141, 634-641.

Hawkins, B.A., Marino, P.C., 1997. The colonization of native phytophagous insects in North America by exotic parasitoids. Oecologia 112, 566-571.

Hopper, K.R., 1998. Assessing and improving safety of introductions for biological control. Phytoprotection 79 (Suppl.), 84-93.

Howarth, F.G., 1991. Environmental impacts of classical biological control. Annu. Rev. Entomol. 36, 485-509.

Julien, M.H., Griffiths, M.W., 1998. Biological Control of Weeds. A World Catalogue of Agents and Their Target Weeds, Fourth ed. CABI Publishing, Oxon, UK.

Julien, M.H., Kerr, J.D., Chan, R.R., 1984. Biological control: an evaluation. Prot. Ecol. 7, 3-25.

Keller, M., 1984. Reassessing evidence for competitive exclusion of introduced natural enemies. Environ. Entomol. 13, 192-195.

Leveque, L., Monge, J., Danielle, R., VanAlebeek, F., Huignard, J., 1993. Analysis of multiparasitism by Eupelmus vuilleti (Craw) (Eupelmidae) and Dinarmus basalis (Rond) (Pteromalidae) in the presence of one of their common hosts, Bruchidius atrolineatus (Pic) (Coleoptera Bruchidae). Oecologia 94, 272-277.

McFadyen, R.E.C., 1998. Biological control of weeds. Annu. Rev. Entomol. 43, 369-393.

Myers, J.H., 1985. How many insect species are necessary for successful biocontrol of weeds? In: Delfosse, E.S. (Ed.), Proceedings of the 6th International Symposium on the Biological Control of Weeds, Agriculture Canada. Canadian Govt. Printing Office, Ottawa, pp. 77-82.

Myers, J.H., Higgins, C., Kovacs, E., 1989. How many insect species are necessary for the biological control of insects? Environ. Entomol. 18, 541-547.

Myers, J.H., Risley, C., 2000. Why reduced seed production is not necessarily translated into successful biological control. In: 
Spencer, N.E. (Ed.), Proceedings of the 10th International Symposium on Biological Control of Weeds. USDA-ARS and Montana State University, Bozeman, MT, USA, pp. 569-581.

Reitz, S.R., 1996. Interspecific competition between two parasitoids of Helicoverpa zea: Eucelatoria bryani and E. rubentis. Entomol. Exp. Appl. 79, 227-234.
Rosenheim, J.A., Kaya, H.K., Ehler, L.E., Marois, J.J., Jaffee, B.A., 1995. Intraguild predation among biological control agents - theory and evidence. Biol. Control 5, 303-335.

SPSS., 1998. SYSTAT 8.0 for Windows. SPSS Inc., Chicago, IL, USA.

Trexler, J.C., Travis, J., 1993. Non-traditional regression analyses. Ecology 74, 1629-1637. 